\title{
Transparent nanocellular PMMA: Characterization and modeling of the optical properties
}

\author{
Judith Martín-de León ${ }^{\mathrm{a}, *}$, Jose Luis Pura ${ }^{\mathrm{b}}$, Victoria Bernardo ${ }^{\mathrm{a}}$, Miguel Ángel Rodríguez-Pérez ${ }^{\mathrm{a}}$ \\ ${ }^{a}$ Cellular Materials Laboratory (CellMat), Condensed Matter Physics Department, Universidad de Valladolid, 47011, Spain \\ ${ }^{\mathrm{b}}$ Gds Optronlab, Condensed Matter Physics Department, Universidad de Valladolid, 47011, Spain
}

\section{H I G H L I G H T S}

- Transmittance to visible light of transparent nanocellular polymethylmethacrylate was measured.

- Samples with a cell size $14 \mathrm{~nm}$ and a relative density of 0.46 can present transmittances as high as 0.94 .

- A theoretical model predicting the transmittance to visible light of nanocellular polymers has been developed.

- Rayleigh scattering is main mechanisms of light transmission in transparent nanocellular polymethylmethacrylate.

\section{A R T I C L E I N F O}

\section{Keywords:}

Transparent

Nanocellular polymer

Modelling

\begin{abstract}
A B S T R A C T
In this work, the optical properties of transparent nanocellular polymethylmethacrylate (PMMA) have been studied, experimental and theoretically. Transmittance measurements of samples presenting different cell sizes $(14,24,39$ and $225 \mathrm{~nm})$ and a constant relative density of around 0.45 have been carried out obtaining values as high as 0.94 for the sample with the smaller cell size and a thickness of $0.05 \mathrm{~mm}$. In addition, the light absorption coefficient has been measured as a function of cell size and wavelength. It has been found that the transmittance has a strong dependence with the wavelength, presenting these transparent materials Rayleigh scattering. On the other hand, the transmission of visible light through these nanocellular materials has been modelled for the first time. The developed model reproduces with good accuracy the trends observed in the experimental results and provides remarkable insights into the physics mechanisms controlling the optical behavior of these materials.
\end{abstract}

\section{Introduction}

Nanocellular polymers are nowadays the spotlight of numerous studies in the field of cellular materials science. This is due to the fact that it has been proved recently that they present new and improved properties in comparison with those presented by conventional or micro cellular materials [1]. Nanocellular polymers have the expected advantages of cellular materials, such as weight reduction and therefore the possibility of saving raw materials [2], excellent stiffness and strength to weight ratio and in addition some improved properties due to their reduced cell size such as a reduced thermal conductivity and a very high surface to volume ratio [3,4]. Besides, nanocellular polymers can present characteristics utterly unknown to date in the cellular polymers field. One of these new characteristics recently discovered is that nanocellular polymers can present a transparent character [5]. All these properties make nanocellular polymers materials with a high potential because they can combine properties never seen together before, being possible to use them in new sectors and possibly in still unknown applications. Some possible applications have been proposed in previous papers such as the possibility of producing super-insulating windows, combining the transparent character of the material and its low thermal conductivity [6]. Additionally, transparent nanocellular polymers could also be used to replace transparent solid polymer films used in many applications, such as the protective layer of electronic device screens, photo frames or in medical applications such as contact lenses. By using a transparent nanocellular polymer instead of a solid film, it would be possible to save in raw material cost and weight due to their reduced density.

In our previous work [5], some transparent nanocellular PMMA samples were produced for the first time using the gas dissolution foaming technique. This goal was accomplished by reaching a very high $\mathrm{CO}_{2}$ solubility in the material. The increase in solubility was obtained by means of reducing up to $-32{ }^{\circ} \mathrm{C}$ the saturation temperature. With this temperature and by changing the saturation pressures from 6 to

\footnotetext{
* Corresponding author.

E-mail address: jmadeleon@fmc.uva.es (J. Martín-de León).
} 
$20 \mathrm{MPa}$, samples with cell sizes from $14 \mathrm{~nm}$ to $39 \mathrm{~nm}$ were obtained and its transparency was demonstrated using optical images. However, although it has been proved that the production of this type of materials is achievable, as far as the authors know, there is only one precedent in which the optical properties of these materials is reported. S. PérezTamarit et al. published the transmittance of a single sample with a cell size of $25 \mathrm{~nm}$ and $1 \mathrm{~mm}$ of thickness [7]. In addition, the modelling of the optical behavior of nanocellular polymers has not been previously reported.

In this work, a detailed characterization of the optical transmittance of a collection of transparent nanocellular PMMA with different cell sizes and thickness is provided, together with a theoretical model that describes the interaction of visible light with this type of nanocellular material.

\section{Materials and methods}

\subsection{Materials}

Pellets of polymethylmethacrylate V825T (PMMA) were supplied by ALTUGLAS ${ }^{\circ}$ International (Colombes, France). The material presents a density of $\rho=1.19 \mathrm{~g} / \mathrm{cm}^{3}$ (measured at $23^{\circ} \mathrm{C}$ and $50 \%$ relative humidity) a glass transition temperature of $T_{g}=114.5^{\circ}$ (measured by DSC) and a molecular weight $M_{n}=43 \mathrm{~kg} / \mathrm{mol}$ and $M_{w}=83 \mathrm{~kg} / \mathrm{mol}$.

Medical grade $\mathrm{CO}_{2}$ (99.9\% purity) was used as the blowing agent for the foaming experiments.

\subsection{Samples preparation}

Solid sheets of PMMA were first produced from the initial pellets by using a hot plate press. Pellets were heated at $250^{\circ} \mathrm{C}$ for $9 \mathrm{~min}$, then the material was pressed under a constant pressure of $35 \mathrm{MPa}$ for an additional minute. Lastly, sheets were cooled down at room temperature under the same pressure. Samples of $75 \times 75 \times 4 \mathrm{~mm}^{3}$ were obtained and then cut to the desired dimensions $\left(30 \times 30 \times 4 \mathrm{~mm}^{3}\right)$ for the foaming experiments.

Gas dissolution foaming process [8] has been used to produce transparent nanocellular PMMA [9]. These foaming tests were carried out in a pressure vessel (model PARR 4760) provided by Parr Instrument Company (Moline, IL, USA) placed inside a freezer that allows reaching temperatures from $-15^{\circ} \mathrm{C}$ to $-32^{\circ} \mathrm{C}$. Moreover, the pressure system comprises a pump (model SFT-10) supplied by Supercritical Fluid Technologies Inc. (Neward, DE, USA). After saturation, samples were foamed in thermal baths (J.P. Selecta Model 600685, Grupo Selecta, Barcelona, Spain).

The processing parameters of the gas dissolution process were chosen according to a previous work [5] with the main objective of obtaining cellular materials with cell sizes below $50 \mathrm{~nm}$ and relatives densities around 0.4 . Saturation temperature was $-32{ }^{\circ} \mathrm{C}$ for all the experiments, while three different saturation pressures were used $(6,10$ and $20 \mathrm{MPa}$ ). Saturation time was 15 days [10] in order to ensure the complete saturation of the samples. After the complete saturation, the pressure was released at a ratio of 10,31 and $75 \mathrm{MPa} / \mathrm{s}$ respectively (the pressure versus time curves and the way to obtain the pressure drop rate is provided in the supporting information (Fig. S1). Foaming parameters were $40^{\circ} \mathrm{C}$ for the foaming temperature and $2 \mathrm{~min}$ for the foaming time. The time between the release of the pressure in the vessel and the foaming of the samples was $1 \mathrm{~min}$ in all experiments.

Moreover, a sample with a cell size closer to the wavelength of visible light (around $200 \mathrm{~nm}$ ) has been produced and used as a reference. A pressure vessel (model PARR 4681) provided by Parr Instrument Company (Moline, IL, USA) was used for this purpose. Process parameters were chosen in order to obtain the desired cell size and relative density, taking into account a previous work [11]. The process parameters were $30 \mathrm{MPa}$ and $25^{\circ} \mathrm{C}$ as saturation parameters and $40^{\circ} \mathrm{C}, 1 \mathrm{~min}$ as foaming parameters. In this case, the depressurization rate was $100 \mathrm{MPa} / \mathrm{s}$ and the desorption time was $3 \mathrm{~min}$.

Finally, thin plane-parallel sheets were obtained from the foamed samples with a precision cutting machine (Mod.1000 IsoMet). Homogenous and uniform samples of various thicknesses (from 0.05 to $1 \mathrm{~mm}$ ) and sizes of $3 \times 3 \mathrm{~cm}^{2}$ were obtained for the transmittance measurements. The surface quality of all the samples were comparable, because all of them were produced using the same method. SEM images of the surface quality of the samples have been include in the supporting information (Fig. S3).

\subsection{Characterization techniques}

\subsubsection{Density}

The density of the solid PMMA $\left(\rho_{\mathrm{s}}\right)$ was determined with a gas pycnometer (Mod. AccuPyc II 1340, Micromeritics, Norcross, GA, USA). For the density of cellular materials $\left(\rho_{f}\right)$, a density determination kit for an AT261 Mettler-Toledo balance was used, the relative density was determined with the water displacement method, based on Archimedes' principle. Before performing this measurement, the solid skins of the samples were removed (more than $200 \mu \mathrm{m}$ on each side). The density of the thin slices used for the optical measurements were the same of that of the bulk sample without solid skins. Relative density $\left(\rho_{r}\right)$ is defined as follows: $\left(\rho_{r}=\rho_{f} / \rho_{s}\right)$.

\subsubsection{Scanning electron microscopy}

Cellular structure was visualized with an ESEM Scanning Electron Microscope (QUANTA 200 FEG, Hillsboro, OR, USA). In order to obtain these images, samples were first cooled in liquid nitrogen to preserve the original cellular structure in the fracture. Then, they were coated with gold by using a sputter coater (model SDC 005, Balzers Union, Balzers, Liechtenstein). In order to characterize the cellular structure, a software based on ImageJ/FIJI [12] was used. The cell nucleation density $\left(\mathrm{N}_{0}\right)$ was determined using the Kumar's theoretical method [13], while the cell size distribution and the average cell size $(\phi)$ was determined by averaging the size of more than 200 cells. Moreover, the standard deviation of the cell size distribution (SD) was determined and finally the parameter $S D / \phi$ was calculated. This parameter allows evaluating the homogeneity of each cell size distribution.

\subsubsection{X-ray radiography}

In order to ensure that the thin sheets of nanocellular materials under study are free of defects, X-ray radiographs were taken by using a home-made-X-ray imaging equipment with $3 \mu \mathrm{m}$ of spatial resolution [14].

\subsubsection{Transmittance measurements}

Transmittance measurements were carried out to determine the transparency of the cellular materials. Transmittance $\left(T=I / I_{0}\right)$ is defined as the ratio between the transmitted intensity reaching the detector $(I)$ and the incident one $\left(I_{0}\right)$. The transmittance of the cellular materials $\left(T_{c}\right)$ were normalized by the transmittance of the solid PMMA $\left(T_{S}\right)$ (this value is around 0.95 for every thickness and wavelength), so the experimental values of transmittance given in this work are defined as $T=T_{c} / T_{s}$.

The device for these transmittance measurements consists of three different lasers (blue: $450 \mathrm{~nm}$, green: $532 \mathrm{~nm}$ and red: $650 \mathrm{~nm}, 10 \mathrm{~mW}$ ) acting as light sources, and a photodiode joined to an integrating sphere with a $12.5 \mathrm{~mm}$ window (PRW0505, Gigahertz-Optik) connected to a photometer (X94, Gigahertz- Optik) working as light detector. Samples are placed directly in the window of the integrating sphere to collect all the light scattered by the samples. The laser-detector distance was $30 \mathrm{~mm}$ fixing the size of the laser beam at the surface of the sample (2 mm). 


\subsection{5. $U V$-VIS}

The dependence of the transmittance with the wavelength was measured using an ultraviolet spectrometer (UV-2102 PC, Shimadzu), that allows measuring the transmittance for wavelengths in the range from $400 \mathrm{~nm}$ to $800 \mathrm{~nm}$. The experiments were performed with an aperture of the slit of $1 \mathrm{~nm}$. The spectrometer was used in the energy mode in order to amplify the signal by a factor of 2. Solid PMMA and nanocellular materials were measured. The energy measured when solid PMMA was used as sample was taken as the incident energy while the measured with the nanocellular samples was considered as the transmitted energy, being the transmittance the ratio between both measurements. Samples were placed as near as possible to the detector, but because of the set-up in this equipment there exists a distance between the sample and the detector (around $2 \mathrm{~cm}$ ), so the transmittance values measured in this equipment cannot be taken as absolute, because some of the transmitted light does not reach the detector. Due to this fact, these measurements were only used to evaluate the effect of wavelength on the transmittance.

\subsubsection{Modeling}

Light transmission process through nanocellular materials has been modeled. The primary objective of this modeling has been calculating the transmittance theoretically. The commercial software COMSOL Multiphysics has been used for the modeling purposes.

First of all, nanocellular PMMA has been modeled; samples are simulated as an infinite plane film. This is performed by starting with a parallelepiped which length in the x-direction has been considered the thickness of the sample. Then, symmetry conditions are applied in the $y$ and $\mathrm{z}$ directions to simulate the infinite plane sample and to maintain the computational cost at a minimum. Nanocellular materials have been modeled as biphasic materials formed by a solid matrix of PMMA (introducing the data of the refractive index $(n=1.49)$ and the dielectric constant $(\varepsilon=2.22)$ of the material, given by the manufacturer) and a second air phase formed by the cells.

As it can be seen in Fig. 1 a, cells have been represented by spheres with fcc packaging (because together with the hexagonal packaging this is the more compact one). The pore size is homogeneous and can be selected as required, the density of the sample is also tuneable by compacting the packing of spheres, but because of a geometric limitation there exists a minimum relative density of around 0.26 , below of which the cell wall disappears, and cells join together. The mesh (Fig. 1b) is defined to be smaller than a tenth of the used wavelength and smaller than the selected cell size, to ensure enough resolution in the calculations. Once the main parameters of the material are selected (thickness, density, and cell size) an automatically generated tetrahedral mesh, for both the solid matrix and pores, was created. This mesh creates more elements in the interfaces between the pores and the solid PMMA, and less in the homogeneous phases, air or solid. This allows reaching a higher resolution with the minimum computational cost.

In order to study the interaction of light with these cellular structures, the module of COMSOL Multiphysics Electromagnetic Waves in Frequency Domain was used. This module solves the Maxwell equations for the propagation of light of a fixed wavelength, travelling in the $x$-direction of the nanocellular polymer. One face of the sample was homogeneously illuminated (Fig. 1c) by a linearly polarized plane wave of the selected wavelength and with $1 \mathrm{~W}$ of power.

Then, the light intensity on the opposite face is calculated, thus obtaining the transmittance. As it can be seen in Fig. 1. c, due to the scattering of light in the medium, the intensity of the electromagnetic wave has been reduced in the area under analysis.

When this process is carried out for materials with different cell sizes and a fixed density and thickness, a curve like that represented in Fig. 2 a (raw T) is obtained. This curve has been corrected in order to obtain a realistic transmittance (curve weighted $\mathrm{T}$ in Fig. 2. a.).

To obtain raw $\mathrm{T}$, it has been considered that samples present a uniform cell size (Fig. 1), which means that all cells in the sample have the same size. This idea is not representing a real cellular material, because they all have a cell size distribution. Moreover, using materials with homogeneous cell sizes leads to resonances. As it can be seen in Fig. 2. a., there are some peaks in the curve raw $\mathrm{T}$ appearing as a result of the coupling of the wavelength with the pore size and the sample length. To solve this artifact, samples with a real cell size distribution must be considered. Cell size distributions of the samples produced experimentally in this work are known, these distributions have been fitted to an asymmetric Gaussian distribution (Equation (1)), where $u$ and $v$ are the width of each side of the distribution, $\phi$ is the cell size and $\bar{\phi}$ is the average cell size. Then, by adjusting the parameters $u$ and $v$ with the known distributions, it has been possible to obtain the cell size distribution for each mean cell size (the results of these fitting are shown in the supporting information, Fig. S5).

$\left\{\begin{array}{l}\exp -\frac{(\phi-\bar{\phi})^{2}}{u^{2}} \text { when } \phi<\bar{\phi} \\ \exp -\frac{(\phi-\bar{\phi})^{2}}{v^{2}} \text { when } \phi>\bar{\phi}\end{array}\right.$

Finally, a convolution between raw $\mathrm{T}\left(T_{\phi}\right)$ and the cell size distributions has been calculated to obatin the weighted curve $\mathrm{T}\left(T_{\bar{\phi}}\right)$.

This convolution is explained in Fig. 3. If the cell size distribution is known, the solution can be obtained using a superposition of the contribution of each cell size. Cells with a size $\Phi 1$ and a transmittance T1 will contribute proportionally to the number of these cells that are present in the cellular material. It means they contribute with their relative frequency f1. This is the same for each cell size so if we know the transmittance for each cell size the transmittance of the whole material can be obtained as the addition of each transmittance multiplied by its contribution, it means multiplied by the relative frequency.

It means that for a given mean cell size, the transmittance has been calculated by using Equation (2). Where $f_{\phi}$ are the normalized relative frequency of the cell size and $T_{\phi}$ is the computed transmittance for each cell size.
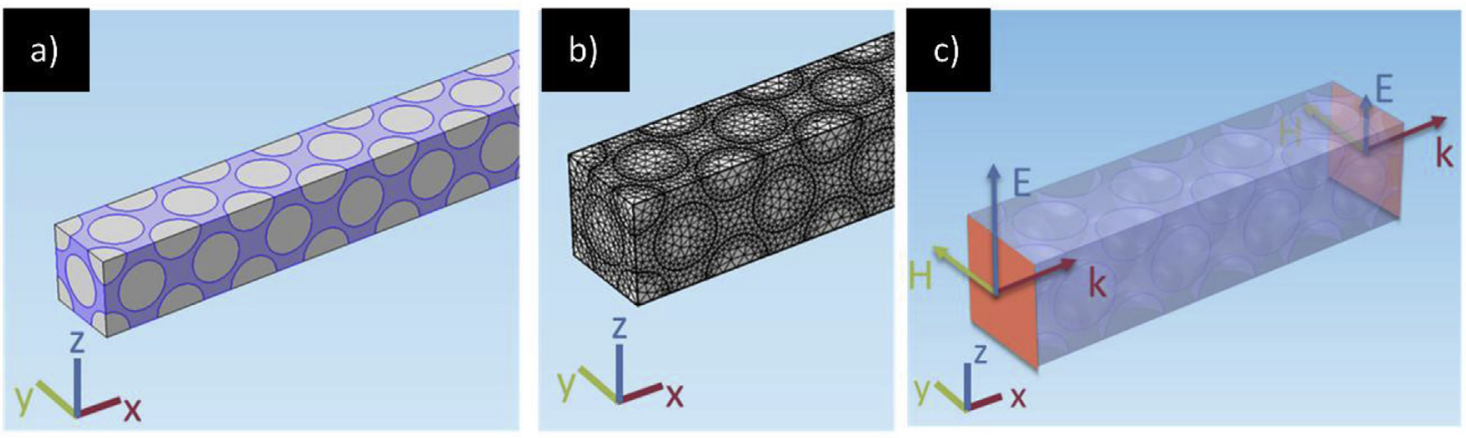

Fig. 1. a) Nanocellular material simulation. b) Meshing of the material. c) Representation of the electromagnetic field. 

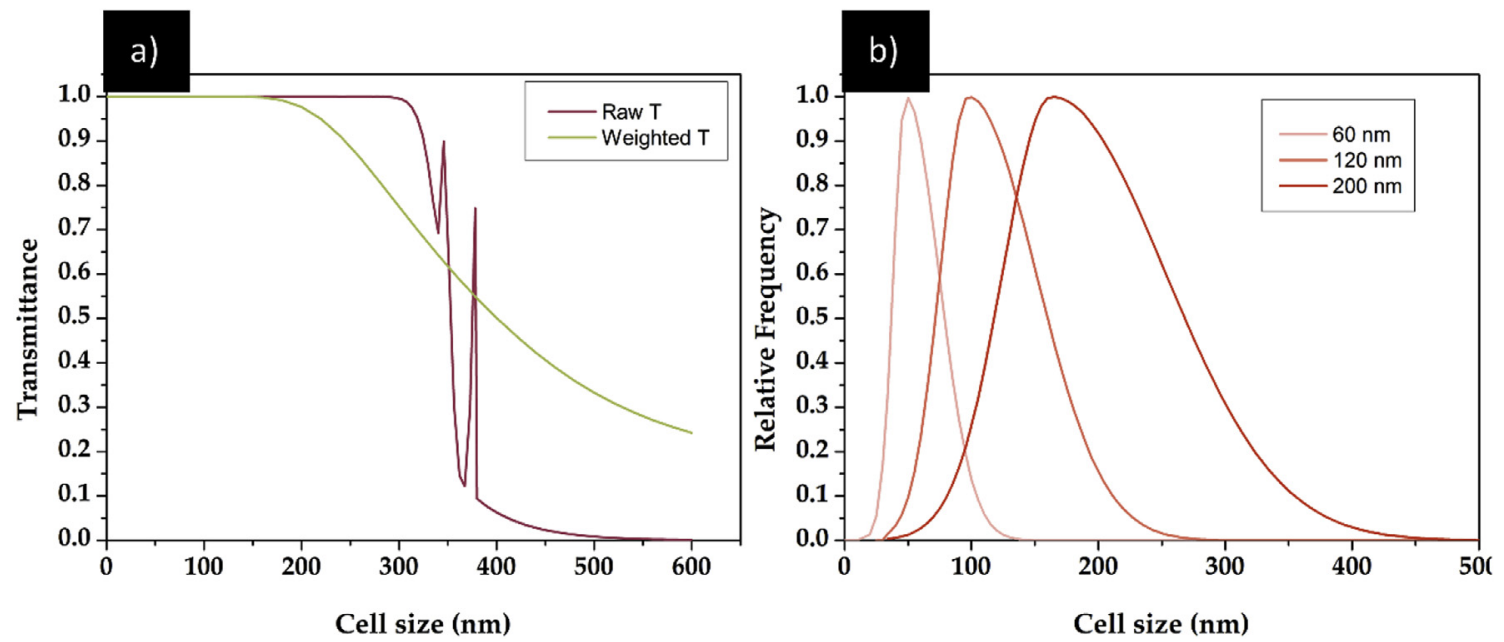

Fig. 2. a) Raw transmittance and Weighted transmittance as a function of the cell size. 2.b) Cell size distributions calculated for different mean cell sizes.

$T_{\bar{\phi}}=\sum_{\phi} f_{\phi} T_{\phi}$

On the other hand, the scattering behaviour of one single cell has been studied. In this case, the model is formed by an infinite bulk PMMA with a single spherical pore at the origin. The model is surrounded by a spherical Perfectly Matched Layers to avoid secondary reflections on the boundaries. The sample is then illuminated by a $532 \mathrm{~nm}$ plane wave traveling in the $\mathrm{x}$-direction and polarized along the $\mathrm{z}$-axis. Once the scattering problem is solved, the far-field output is studied to understand the scattering behaviour as a function of the pore diameter.

\section{Results}

\subsection{Cellular structure}

As it was presented in our previous works $[5,11]$ the use of $30 \mathrm{MPa}$ and $25^{\circ} \mathrm{C}$ as saturation conditions lead to solubilities around $31 \%$, while the use of low saturation temperatures $\left(-32^{\circ} \mathrm{C}\right)$ allows reaching solubilities of $\mathrm{CO}_{2}$ as high as $48 \%$. This results in a change in the cell size from $200 \mathrm{~nm}$ when the saturation temperature is $25^{\circ} \mathrm{C}$ to cell sizes below $50 \mathrm{~nm}$ when saturation temperature is below zero. In this work, in order to produce materials with different cell sizes, three different saturation pressures were used, $(6,10$ and $20 \mathrm{MPa})$, while fixing the
Table 1

Cell nucleation density, cell size and SD/ $\Phi$ for the produced samples.

\begin{tabular}{lllll}
\hline Saturation conditions & $\begin{array}{l}\text { Relative } \\
\text { density }\end{array}$ & $\begin{array}{l}\mathrm{N}_{0} \text { (nuclei/ } \\
\mathrm{cm}^{3} \text { ) }\end{array}$ & $\begin{array}{l}\text { Cell size }(\Phi) \\
(\mathrm{nm})\end{array}$ & $\mathrm{SD} / \phi$ \\
\hline $30 \mathrm{MPa} 25^{\circ} \mathrm{C}$ & 0.47 & $1.8 \cdot 10^{14}$ & 213 & 0.45 \\
$6 \mathrm{MPa}-32{ }^{\circ} \mathrm{C}$ & 0.47 & $7.7 \cdot 10^{15}$ & 39 & 0.35 \\
$10 \mathrm{MPa}-32{ }^{\circ} \mathrm{C}$ & 0.46 & $1.2 \cdot 10^{16}$ & 24 & 0.28 \\
$20 \mathrm{MPa}-32^{\circ} \mathrm{C}$ & 0.46 & $4.0 \cdot 10^{16}$ & 14 & 0.22 \\
\hline
\end{tabular}

saturation temperature at $-32^{\circ} \mathrm{C}$. Afterward, all samples were foamed at $40^{\circ} \mathrm{C}$ for $2 \mathrm{~min}$. All these process conditions lead to samples with the characteristics shown in Table 1. SEM images can be found in the supplementary information (Fig. S2).

As it can be seen, samples with a similar relative density (between 0.46 and 0.47 ) but different cellular structures have been obtained by using the previously described conditions. Thus, samples with cell sizes from $213 \mathrm{~nm}$ to $14 \mathrm{~nm}$ and with cell nucleation densities from $1.8 \cdot 10^{14}$ nuclei/ $\mathrm{cm}^{3}$ to $4.0 \cdot 10^{16}$ nuclei $/ \mathrm{cm}^{3}$ respectively are available for the transmittance experiments. It is also important to notice the change in the parameter $S D / \phi$ indicating the width of the cell size distribution. It is noticed that as cell size becomes smaller, the cell size distribution becomes clearly narrower.
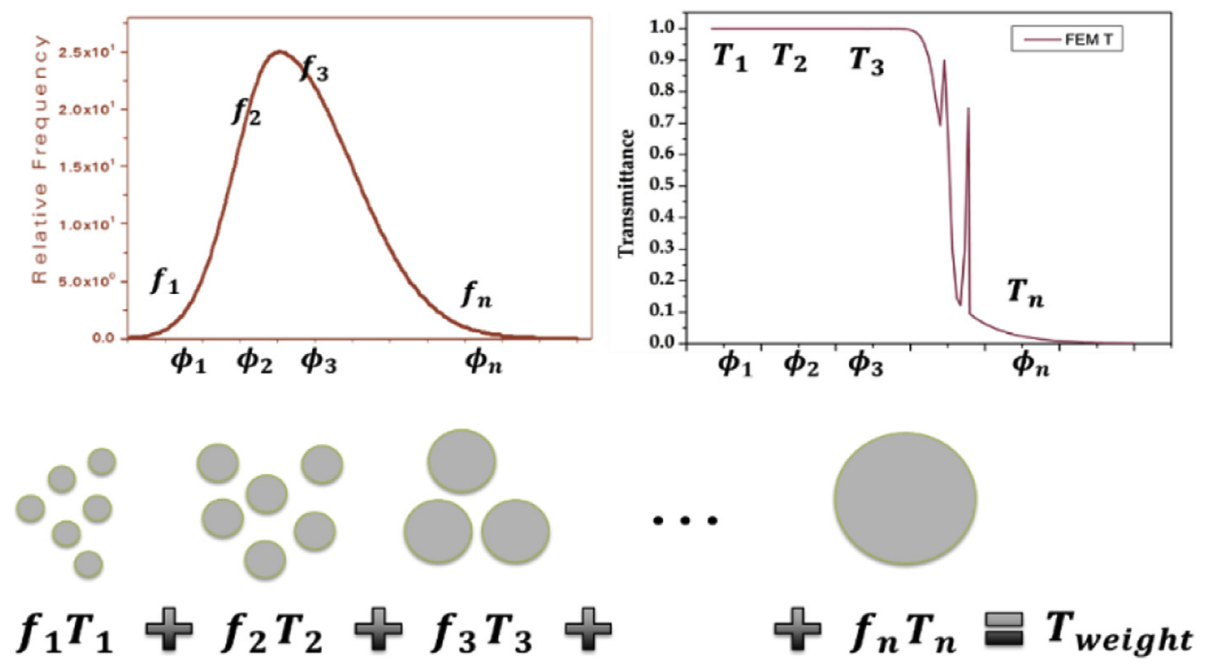

Fig. 3. Explanation of how the transmittance for a given cell size is combined with the cell size distributions. 

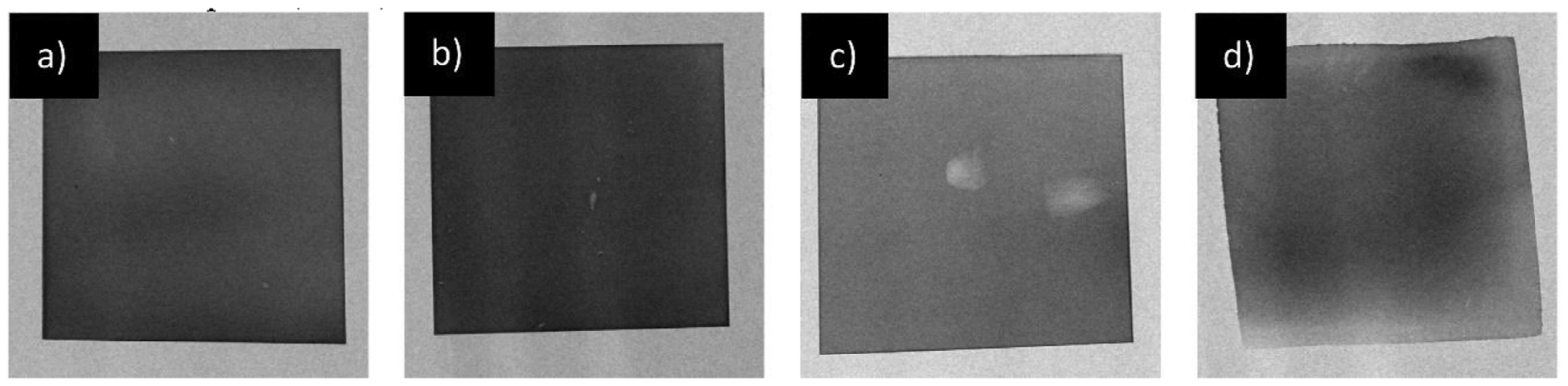

Fig. 4. X-Ray radiography of different samples.

\section{2. $X$-ray radiographs}

Radiographies of all samples were taken to select homogeneous samples free of defects for the characterization of the optical properties. Fig. 4 shows some examples. Samples a) and b) are completely homogeneous while c) has some defects and d) has zones with different opacity (i.e., different density and/or thickness). Samples behaving as samples c) and d) were not used in the optical measurements, taking into account only those samples showing radiographies similar to those of samples a) or b).

\subsection{Transmittance}

\subsubsection{Cell size dependence}

In this section, the results obtained using the green laser $(532 \mathrm{~nm})$ are presented and discussed. The resulted trends with the red and blue lasers were similar. (see supporting information).

Table 2 shows the transmittance results for samples with different cell sizes and thickness. The change between cellular PMMA with $225 \mathrm{~nm}$ of cell size and those with cell sizes below $50 \mathrm{~nm}$ is evident. While the first sample is almost opaque to green light, showing a transmittance near zero even for a thickness as low as $0.05 \mathrm{~mm}$, the values for the rest of the samples are significantly higher. In particular, at low thickness, some of the samples present values higher than 0.90 .

In addition, the change between the three samples with less than $50 \mathrm{~nm}$ is remarkable. Sample with a mean cell size of $14 \mathrm{~nm}$ reach values of transmittance as high as 0.94 with $0.05 \mathrm{~mm}$ of thickness, while for the $39 \mathrm{~nm}$ sample this value is reduced down to 0.88 . In addition, when the thickness increases up to $1 \mathrm{~mm}$ transmittance values become smaller, but not at all negligible. For the $14 \mathrm{~nm}$ sample, the transmittance is still 0.5 for $1 \mathrm{~mm}$ in thickness, which is a high value in comparison with the one presented by the sample with a cell size of $225 \mathrm{~nm}$.

According to scattering theories, the maximum particle size for the light to pass through is one-tenth of the wavelength [15]. This means that for a wavelength of $532 \mathrm{~nm}$ the maximum cell size for the sample to be transparent is around $50 \mathrm{~nm}$. This is in accordance with the showed results, where only samples below $50 \mathrm{~nm}$ have a significant transmittance. Moreover, the cell size distribution needs to be considered to understand the results, the sample with a cell size of $39 \mathrm{~nm}$ has a broader cell size distribution than the sample with a cell size of $14 \mathrm{~nm}$, that means that in the sample with a higher mean cell size there

Table 2

Transmittance of samples with different cell size and thickness for a wavelength of $532 \mathrm{~nm}$.

\begin{tabular}{llllll}
\hline $\begin{array}{l}\text { Cell } \\
\text { size } \\
(\mathrm{nm})\end{array}$ & $\begin{array}{l}\text { Thickness } \\
0.05 \mathrm{~mm}\end{array}$ & $\begin{array}{l}\text { Thickness } \\
0.1 \mathrm{~mm}\end{array}$ & $\begin{array}{l}\text { Thickness } \\
0.2 \mathrm{~mm}\end{array}$ & $\begin{array}{l}\text { Thickness } \\
0.5 \mathrm{~mm}\end{array}$ & $\begin{array}{l}\text { Thickness } \\
1 \mathrm{~mm}\end{array}$ \\
\hline 225 & 0.09 & 0.06 & 0.04 & 0.02 & 0.01 \\
39 & 0.88 & 0.81 & 0.74 & 0.53 & 0.36 \\
24 & 0.92 & 0.85 & 0.84 & 0.56 & 0.41 \\
14 & 0.94 & 0.92 & 0.83 & 0.66 & 0.48 \\
\hline
\end{tabular}

are some cells with sizes higher than $50 \mathrm{~nm}$ that are reducing the total transmittance.

In conclusion, to maximize the transmittance of the cellular materials, it is essential to minimize the cell size as well as narrowing the cell size distribution.

\subsubsection{Dependence of the transmittance with the thickness}

By representing $\operatorname{Ln}(T)$ as a function of the thickness Fig. 5, it can be seen that the relationship between the transmittance and the thickness (l) of the sample follow an exponential behaviour:

$T=e^{-\mu l}$

where $\mu$ is a coefficient that can be related with the light that it not passing through the material.

The obtained results for a wavelength of $532 \mathrm{~nm}$ are presented in Fig. 5, from which it can be deduced that transmittance has an exponential behaviour with the thickness in all the samples under study. It means that the $\mu$ coefficient can be then obtained from the slope of the curve of each material, being possible to determine the value of transmittance for any thickness. Moreover, large differences between the samples have been obtained, showing that $\mu$ increases as cell size becomes higher (Fig. 5b). In accordance with what has been discussed so far, this fact shows that as cell size increases, less light can pass through the sample. The results for the other wavelengths followed the same trends and have been included in the supporting information (Fig. S4).

\subsubsection{Wavelength dependence}

The effect of the light wavelength on the transmittance of the samples was studied in the samples of $0.2 \mathrm{~mm}$ in thickness by means of a UV-VIS spectrometer. A continuous transmittance curve from $400 \mathrm{~nm}$ to $800 \mathrm{~nm}$ of wavelength was obtained. Fig. 4 shows the results for the samples with cell sizes smaller than $50 \mathrm{~nm}$. The results for the sample with a cell size of $225 \mathrm{~nm}$ are not given because of the very small transmittance of these samples that made impossible detecting light by the UV-VIS equipment used in this research.

As it can be seen in Fig. 6 a, the wavelength has a strong effect on the transmittance of the samples; small wavelengths are less likely to travel through the samples than the higher ones. It means the red light goes across the cellular material easily than blue light. This effect has been previously observed in transparent silica aerogels [16], and these materials are similar to the nanocellular PMMA produced in this work as far as cell size and transparency of the base material are concerned. This strong wavelength dependence was attributed to Rayleigh scattering in previous works studying this effect in aerogels [16-19]. Rayleigh scattering occurs when the light interacts with objects smaller than about a tenth of the wavelength. The intensity of the scattered light depends, according to Rayleigh law, on $\lambda^{-4}$, and the transmittance can be calculated according to Equation (4) [20]:

$T=A e^{-\frac{B l}{\lambda^{4}}}$

Where A and B are constants. The $\operatorname{Ln}(T)$ as a function of $l / \lambda^{4}$ has been 

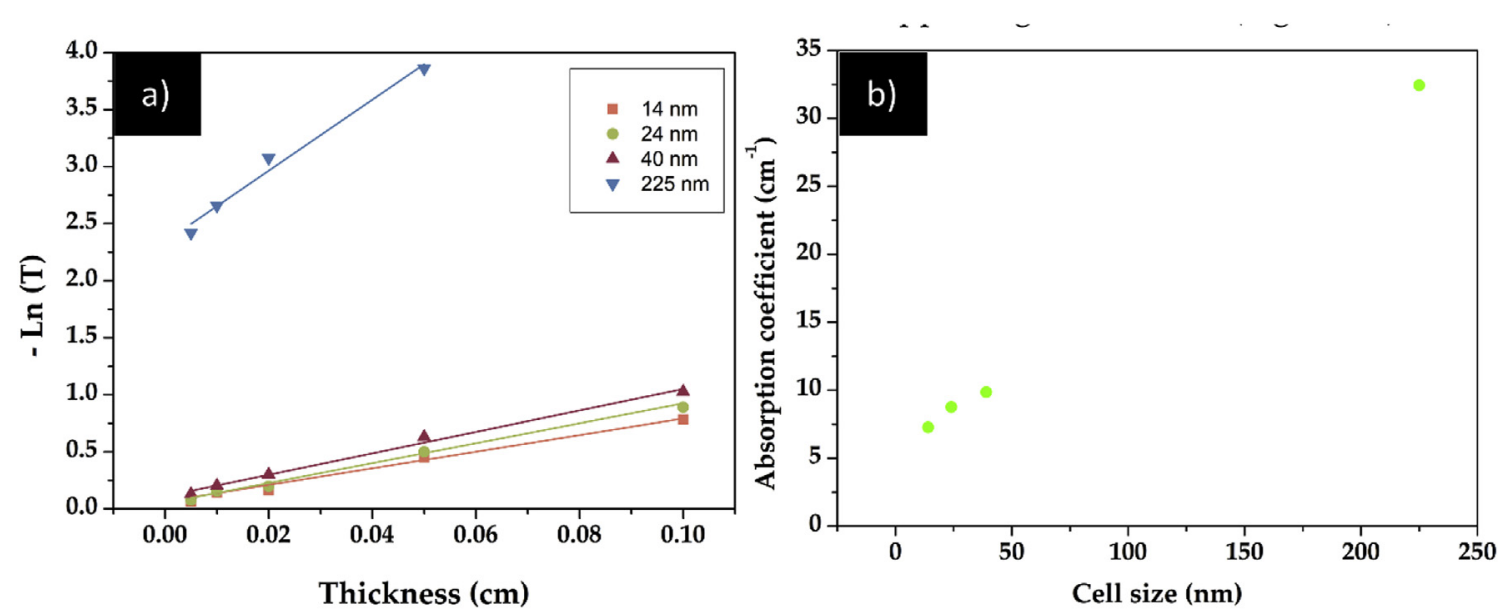

Fig. 5. a) Exponential fitting for the experimental results for a wavelength of $532 \mathrm{~nm}$ b) Absorption coefficient as a function of cell size for a wavelength of $532 \mathrm{~nm}$.

presented in Fig. 6b. As it can be observed, straight lines are obtained which means that the nanocellular PMMA samples with cell sizes below $50 \mathrm{~nm}$ present Rayleigh scattering as it was previously demonstrated for silica aerogels.

\subsection{Modeling}

\subsubsection{Cell size dependence}

The transmittance as a function of the cell size has been modelled for cell sizes from 5 to $250 \mathrm{~nm}$. This modeling has been carried out for a relative density of 0.47 and by fixing a sample thickness of $1.22 \mu \mathrm{m}$. This sample thickness has been chosen because it is the maximum that can be solved with the necessary precision and affordable computation times. So as to obtain the transmittance for different thicknesses Equation (5), derived from Equation (3), was used. Where $T_{0}$ is the calculated transmittance for an initial thickness $l_{0}$ and $T$ is the transmittance for a new thickness $l$.

$T=T_{0}^{\frac{l}{l_{0}}}$

This equation allows obtaining the transmittance for any thickness from the calculated data. The results obtained for four different thicknesses (from 0.1 to $1 \mathrm{~mm}$ ) and for a wavelength of $532 \mathrm{~nm}$ are shown in Fig. $7 \mathrm{~b}$ The tendency with the cell size is clear: below $50 \mathrm{~nm}$ the transmittance is high, while when this value of cell size becomes higher, the transmittance sharply decreases up to reach 0 for cells between 125 and $200 \mathrm{~nm}$ depending of the sample thickness.

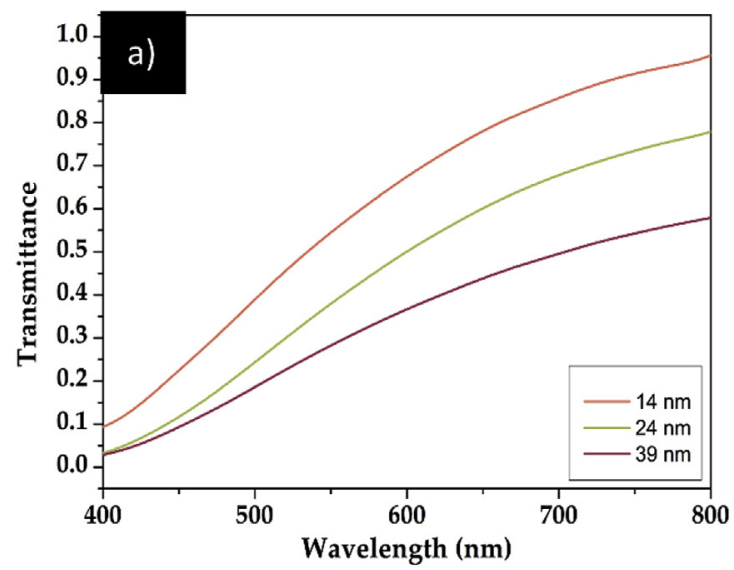

The experimental results are compared with the results from the modelling in Fig. 6. b. The results show that both, experimental and theoretical data, clearly fit and show the same trends, which demonstrates that the proposed model can predict the transmittance values.

Also, using Comsol Multiphysics, a representation of the special distribution of the modulus of the electric field can be obtained for samples with different cell sizes (Fig. 7a). The results for a sample with a cell size of $225 \mathrm{~nm}$ and a sample with a cell size of $39 \mathrm{~nm}$ are included in this figure. Differences between samples are clear, scale bars of the intensity of the electric field $(\mathrm{V} / \mathrm{m})$ are shown in the figure, these values cannot be taken as absolutes because they should be compared with the initial intensity, but they can be used qualitatively.

When the pore size is small enough, the electromagnetic field is allowed to travel through the cells, and the intensity inside the cells and in the solid phase is similar. It seems that the electromagnetic field is traveling in a continuous way through the material and the scattering due to the presence of the cells is negligible. While when the pore size is larger than $100 \mathrm{~nm}$, the electromagnetic field cannot enter the cells, the electromagnetic field is only traveling through solid PMMA, and the scattering is much higher.

\subsubsection{Wavelength dependence}

In order to study the variation of the transmittance with the wavelength, samples with a relative density of 0.47 and different cell sizes have been modelled, by varying the wavelength from 400 to $800 \mathrm{~nm}$. When this modeling was carried out resonances like those shown in the previous section were observed. To overcome this problem, samples

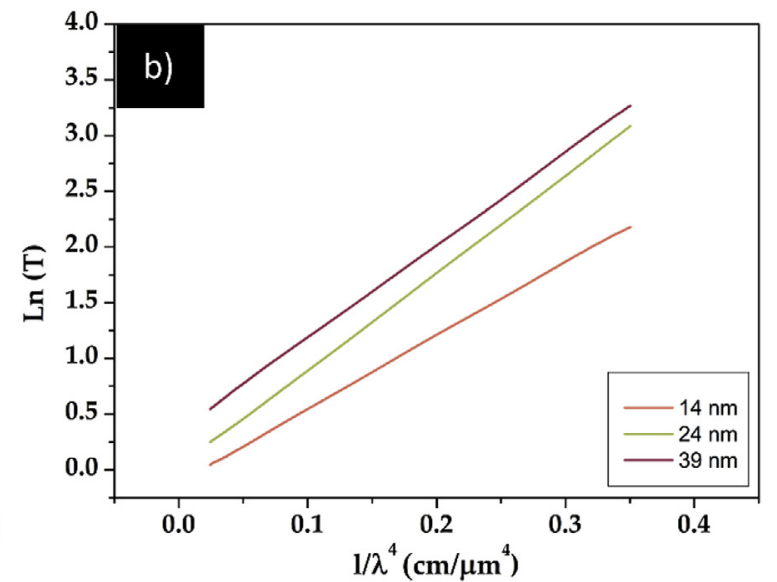

Fig. 6. a) Transmittance as a function of the wavelength for the three samples with cells sizes smaller than $50 \mathrm{~nm}$. b) Linearization of equation (4) to observe the $\lambda^{-4}$ dependence. 

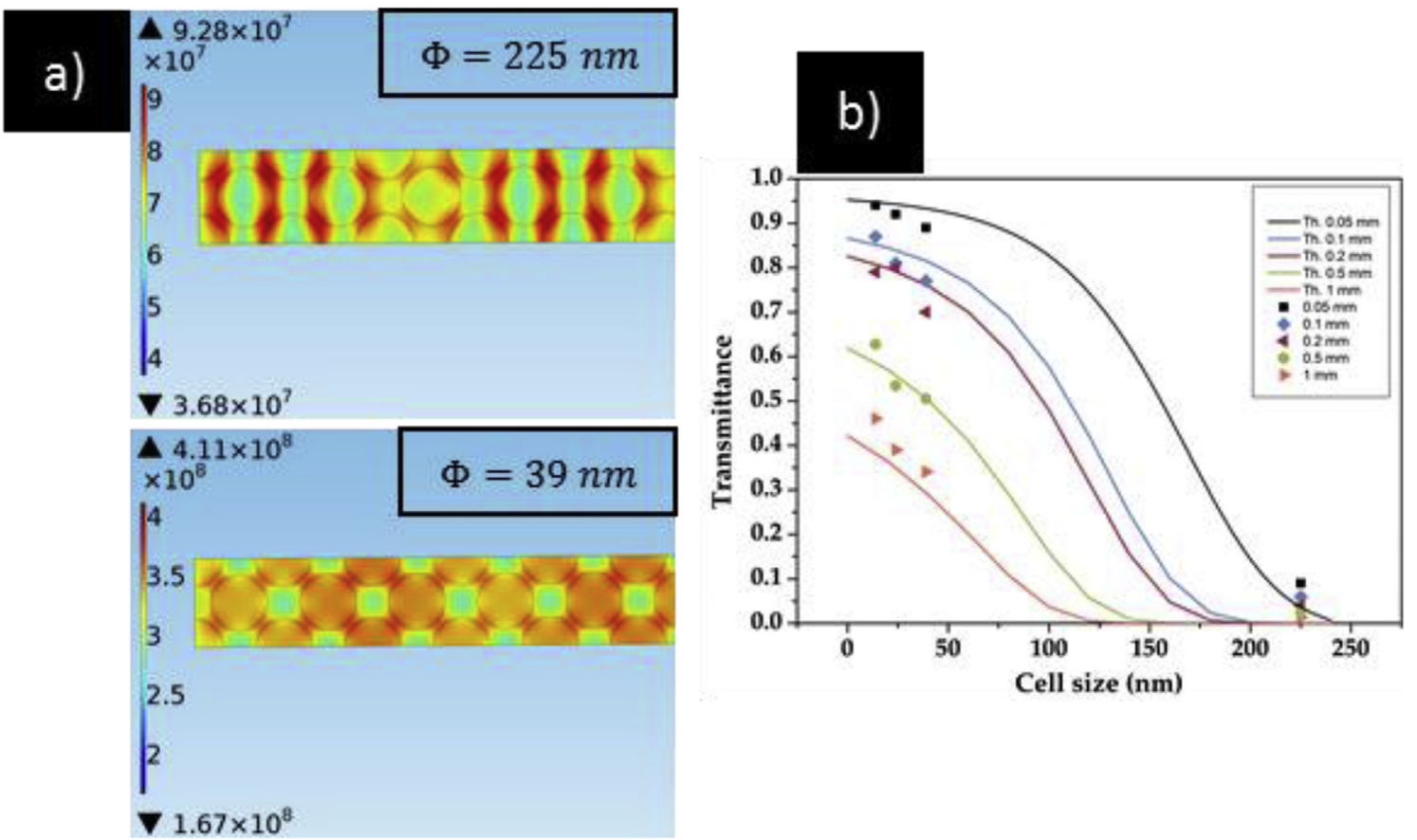

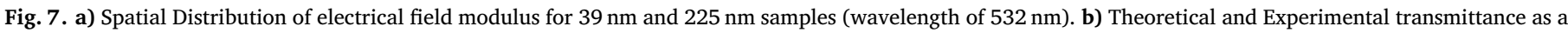
function of cell size (wavelength of $532 \mathrm{~nm}$ ).

with different lengths from $1 \mu \mathrm{m}$ to $1.4 \mu \mathrm{m}$ (in steps of $0.05 \mu \mathrm{m}$ ) were considered to eliminate the resonances and then an average of the transmittance was calculated. The refractive index and dielectric constants were considered here as constants because the minimum change they have in the range under study did not introduce any change in the transmittance values.

Fig. 8 shows both a graph transmittance vs. wavelength and images showing the electric field distribution for a sample of cell size $39 \mathrm{~nm}$ and $0.1 \mathrm{~mm}$ in thickness. Obtained results verify what it was observed in the experimental section, there exists a strong dependence of the transmittance with the light wavelength. As it can be seen, the electromagnetic field is not allowed to enter the cells, suffering a strong scattering along the sample when using an incident light of $400 \mathrm{~nm}$. However, this effect is not observed with the $800 \mathrm{~nm}$ light. In this case, light travels from cell to cell and scattering is not discernible. This results in a much higher transmittance at higher wavelengths than at smaller ones.

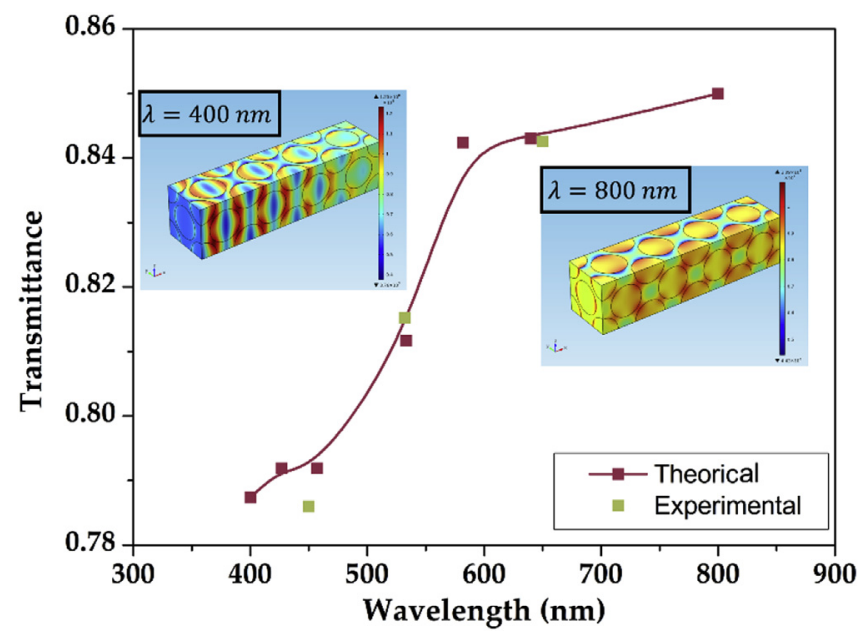

Fig. 8. Theoretical and experimental results of the transmittance as a function of wavelength together with two images showing the electric field modulus.
In addition, these data have also been compared with the experimental ones. In this section, the experimental data obtained by means of the three different lasers are used. As it is observed in Fig. 8, the model reproduces the behaviour experimentally obtained, validating once again the proposed model.

To fully understand the physics behind the obtained results, an additional simulation was carried out. A unique air cell surrounded by PMMA was modeled and then illuminated with linearly polarized light with a traveling in the $\mathrm{x}$-direction and polarized in the $\mathrm{z}$-direction of a wavelength of $532 \mathrm{~nm}$, then the behaviour of light depending on the cell size was studied. Fig. 9. a shows a far-field diagram, i.e., a polar plot of the intensity of the radiation field, that is, the light scattered by the system in each direction. In the diagram, $0^{\circ}$ corresponds to the light scattered in the forward direction (x-positive-axis) while $180^{\circ}$ corresponds to backscattered light. Large differences between cell sizes are appreciable; on the one hand, the amount of scattered light decreases abruptly when cell size becomes smaller, on the other hand, the scattering mechanism is also different for different cell sizes.

These differences result in visible effects when the light passes through real samples (Fig. 9b). The results shown in Fig. 9 reinforce the discussion above; when a cell is clearly smaller than the wavelength, the cell acts as an electric dipole for the electromagnetic field, as it can be seen two lobes appear in the far-field diagram, which is the typical behaviour of a dipole. This effect is known as the Rayleigh effect. Moreover, the amount of scattered light is very small in comparison with higher pores. This leads to a real transparent sample that when the light illuminates allow most of the light passing through it and the small amount that it is Rayleigh-scattered make the sample to acquire a bluish tone while the light passing through becomes red (this is due to the strong dependence of wavelength of this type of scattering). When pores start to be larger but still comparable to the wavelength Mie scattering takes part, the pore acts then like a dipole deformed in the forward direction. In addition, the amount of scattered light is very high resulting in an opaque sample (Fig. 9b).

\section{Conclusions}

Transparent nanocellular PMMA with cell sizes from $14 \mathrm{~nm}$ to 

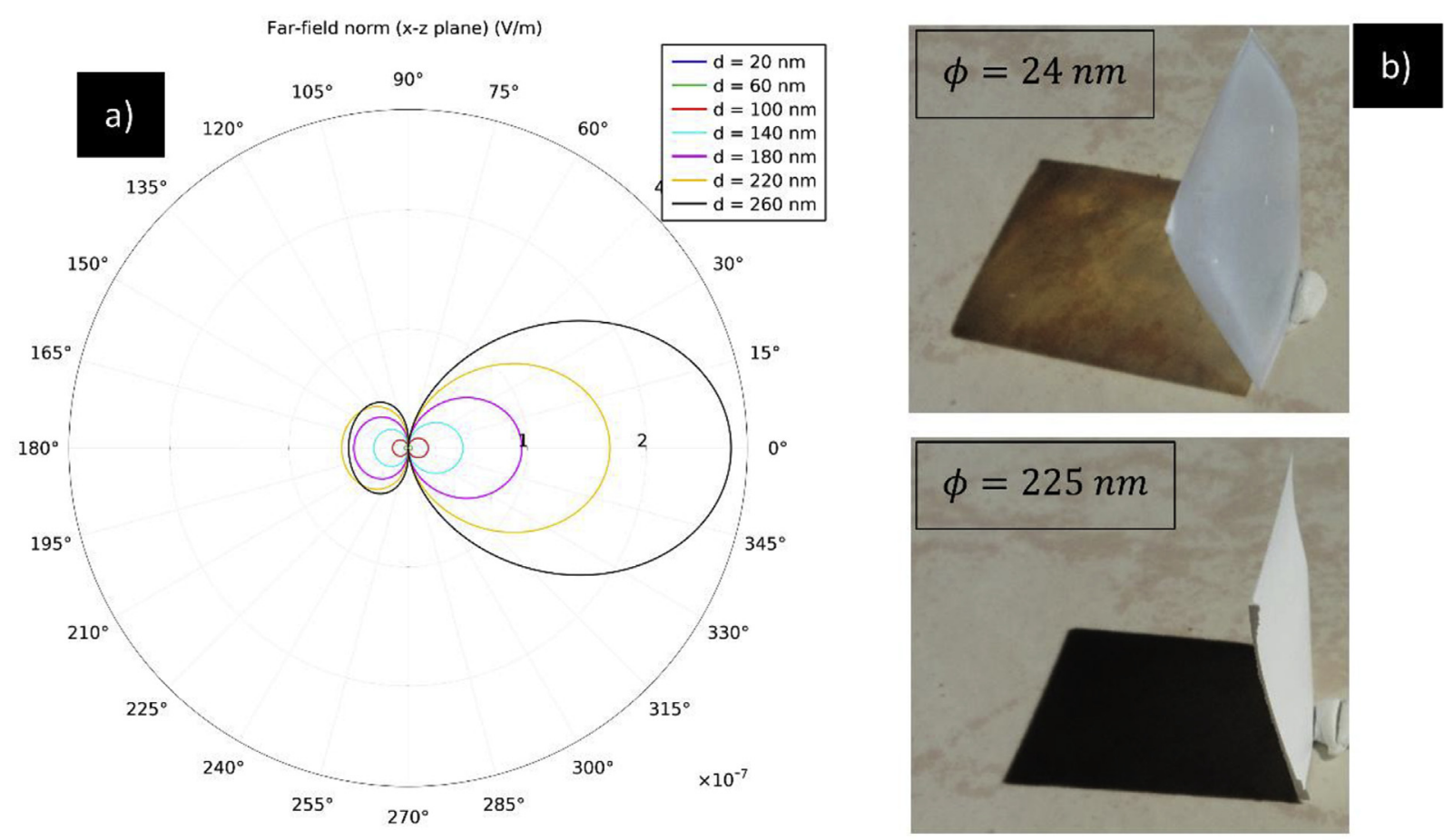

Fig. 9. a) Far-field diagram of a unique pore surrounded by PMMA illuminated with light with a wavelength of $532 \mathrm{~nm}$. b) optical images of the transmission of light through real samples with the same relative density and different cell sizes.

$39 \mathrm{~nm}$ and relative densities around 0.47 have been optically characterized. The transmittance of these materials has been compared with the one of an opaque sample with a cell size of $225 \mathrm{~nm}$. It has been found that while the $225 \mathrm{~nm}$ sample presents values of transmittance near zero for all the used thickness, transmittances as high as 0.94 are achievable when the cell size is $14 \mathrm{~nm}$, and the thickness of the sample is $0.05 \mathrm{~mm}$. Moreover, this sample is able to maintain a transmittance near 0.5 when the thickness of the sample is $1 \mathrm{~mm}$. On the other hand, the dependence on the wavelength was determined to be $\lambda^{-4}$, presenting these transparent materials Rayleigh scattering.

All this has been theoretically modelled; the model was able to predict the trends showed by the experimental results. The presence of Rayleigh scattering has also been predicted by this model. Therefore, we can conclude that for the first time, a theoretical model allows understanding the mechanisms taking place in the transmission of visible light in nanocellular polymers. This model allows calculating the transmittance for any cell size, thickness, and density and for any visible wavelength.

\section{Acknowledgments}

Financial support from the Junta of Castile and Leon grant (J. Martín-de León), FPU grant FPU14/00916 (J.L. Pura) and FPU grant FPU14/02050 (V. Bernardo) from the Spanish Ministry of Education are gratefully acknowledged. Financial assistance from MINECO, FEDER, UE (MAT2015-69234-R) and the Junta de Castile and Leon (VA275P18) are gratefully acknowledged.

\section{Appendix A. Supplementary data}

Supplementary data to this article can be found online at https:// doi.org/10.1016/j.polymer.2019.03.010.

\section{References}

[1] B. Notario, J. Pinto, M.A. Rodriguez-Perez, Prog. Mater. Sci. 78-79 (2016) 93-139.

[2] N.J. Mills, Polymer 34 (1993) 2237.

[3] D. Miller, V. Kumar, Polymer 52 (2011) 2910-2919.

[4] B. Notario, J. Pinto, E. Solorzano, J.A. De Saja, M. Dumon, M.A. Rodriguez-Perez, Polym. (United Kingdom) 56 (2015) 57-67.

[5] J. Martín-de León, V. Bernardo, M.Á. Rodríguez-Pérez, Macromol. Mater. Eng. 3 (2017) 1700343.

[6] H. Guo, V. Kumar, Polymer 56 (2015) 46-56.

[7] S. Pérez-Tamarit, B. Notario, E. Solórzano, M.A. Rodriguez-Perez, Mater. Lett. 210 (2018) 39-41.

[8] I. Singh, A. Gandhi, M. Biswal, S. Mohanty, S.K. Nayak, Cell. Polym. 37 (2018) 121-149.

[9] V. Kumar, N.P. Suh, Polym. Eng. Sci. 30 (1990) 1323-1329.

[10] H. Guo, V. Kumar, Polymer 57 (2015) 157-163.

[11] J. Martín-de León, V. Bernardo, M. Rodríguez-Pérez, Polymers 8 (2016) 265.

[12] J. Pinto, E. Solorzano, M. a. Rodriguez-Perez, J. a. de Saja, J. Cell. Plast. 49 (2013) $555-575$.

[13] V. Kumar, N.P. Suh, Polym. Eng. Sci. 30 (1990) 1323-1329.

[14] E. Solórzano, J. Pinto, S. Pardo, F. Garcia-Moreno, M.A. Rodriguez-Perez, Polym. Test. 32 (2013) 321-329.

[15] D.J. Lockwood, R. Luo (Ed.), Encycl. Color Sci. Technol, Springer Berlin Heidelberg, Berlin, Heidelberg, 2014, pp. 1-12.

[16] A.A. Pisal, A. Venkateswara Rao, J. Porous Mater. 24 (2017) 685-695.

[17] A.J. Hunt, P. Berdahl, Mater. Res. Soc. Symp. Proceeding 32 (1984) 275-280.

[18] A. Soleimani Dorcheh, M.H. Abbasi, J. Mater. Process. Technol. 199 (2008) 10-26.

[19] S.B. Riffat, G. Qiu, Int. J. Low Carbon Technol. 8 (2013) 1-6.

[20] Wanqing Cao, A.J. Hunt, J. Non-Cryst. Solids 176 (1994) 18-25. 